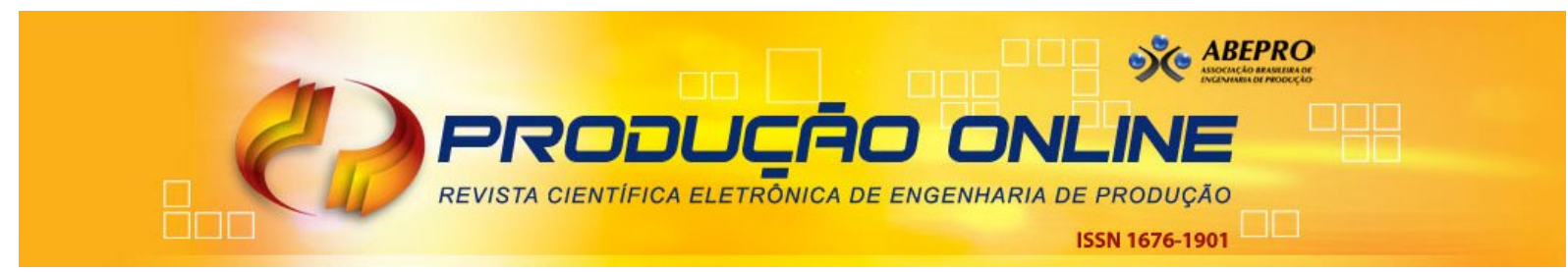

\title{
TRADE-OFFS DE CUSTOS LOGÍSTICOS: UMA AVALIAÇÃO DE CONHECIMENTO EM UMA MULTINACIONAL
}

\section{TRADE-OFF IN LOGISTICS COSTS: AN KNOWLEDGE ASSESSMENT IN A MULTINATIONAL}

\author{
José Alan Barbosa da Silva* E-mail: js.josealan@hotmail.com \\ Fabricio Schlag* E-mail: fabricioschlag@gmail.com \\ Solange da Silva** E-mail: solansilva.ucg@gmail.com \\ *Universidade Estadual de Goiás (UEG), Senador Canedo,GO \\ **Pontifícia Universidade Católica de Goiás (PUC/GO), Goiânia, GO
}

\begin{abstract}
Resumo: Este artigo objetivou verificar a extensão com que os profissionais de logística verificam e analisam seu custo total e os níveis de serviços e se conhecem seus trade-offs. A metodologia compreende revisão bibliográfica e um estudo de caso realizado em uma multinacional, uma das maiores empresas mundial de fragrâncias, cosméticos, beleza e maquiagem. Os dados obtidos demonstraram que os profissionais de logística têm ciência de que, a logística gera impacto econômicofinanceiro na organização. Quanto a experiência e a educação formal, evidenciou-se que, favorecem positivamente a identificação de problemas e a eliminação de parte dos erros e retrabalhos, porque amplia a base de sabedoria dos profissionais. Referente a informações contábeis e logísticas, comprovou-se a falta de informações adequadas e suficientes para a gestão logística da organização. Conclui-se que os profissionais, precisam receber informações logísticas e contábeis que auxiliarão na tomada de decisão e busca do nível de serviço ótimo versus custo total mínimo.
\end{abstract}

Palavras chave: Custos logísticos. Informações contábeis. Níveis de serviço. Trade-off.

Abstract: This study aimed to verify the extent to which logistics professionals verify and analyze their total cost and the service levels and if know their trade-offs. The methodology comprises a bibliographical review and a case study conducted in a multinational, one of the largest world companies of fragrances, cosmetics, beauty, and makeup. The obtained data showed of the logistics professionals are aware that logistics generates economic-financial impact on the organization. Regarding the experience and the formal education, evidencing that the favor positively the identification of problems and the elimination of some of the errors and reworked because it broads the wisdom base of professionals. Concerning to the accounting and logistical information, which proves, the lack of adequate and enough information for the logistics management of the organization. It is concluded that professionals need to receive logistics and accounting information that will aid in the decision making and search for great service levels versus minimum total cost.

Keywords: Logistic costs. Accounting information. Service levels. Trade-off.

\section{INTRODUÇÃO}

Atualmente a logística ganhou destaque no cenário empresarial e passou a ser objeto de estudo de diversos administradores, engenheiros e especialistas em processos e gestão. Sua missão é conquistar novos clientes e atendê-los de forma a 
superar suas expectativas, criando valor para a organização, ainda mais com o advento da globalização, a logística passou a ser considerada como atividade que possibilita se diferenciar dos concorrentes, podendo gerar vantagens competitivas para a organização (DA SILVA, 2018).

Considerando esta visão, a logística exerce papel relevante, sendo a principal responsável para que o produto certo esteja no lugar certo, no tempo certo, na quantidade certa, nas condições certas, a um preço justo e com as informações necessárias (MENTZER, FLINT e HULT, 2001; DA SILVA, 2018; DA SILVA e SILVA, 2018).

Dado a relevância da logística, e conforme enfatizado por Lalonde (1993), e Da Silva (2018), de que não adiantaria um produto com qualidade, bem concebido, com ótima embalagem e ampla promoção, estar no lugar errado, e/ou indisponível quando desejado pelo cliente. Neste contexto, para que seus produtos e serviços sejam disponibilizados aos mercados consumidores com maior qualidade e menor custo, a contribuição prática se dará através da análise das melhores alternativas, contribuindo para o processo de tomada de decisão, fazendo com que os profissionais de administração em logística, passem a conhecer, gerenciar e controlar de forma eficiente os trade-offs (trocas compensatórias) de custos e níveis de serviços, adequando seus esforços para que o atendimento ao cliente ocorra ao menor custo total possível (DA SILVA, 2018).

Dessa forma, Bio, Robles e Faria (2003) e da Silva (2018), advertiram que ao buscar-se atender as especificações dos clientes, com exigências cada vez maiores de nível de serviço, os custos logísticos tendem a aumentar, sendo esse o grande desafio dos profissionais de logística, agregar valor ao cliente por meio de um nível de serviço de excelência, mas, ao menor custo total possível. Sendo assim, a logística impacta na receita, no custo, na utilização e/ou imobilização de capital (CHRISTOPHER e RYALS, 1999; WALTERS, 1999; BALLOU, 2006; PRESUTTI e MAWHINNEY, 2007; AMARAL e GUERREIRO, 2014; DA SILVA, 2018; DA SILVA e SILVA, 2018, DA SILVA e SCHLAG, 2017).

Neste sentido, tornar-se evidente que é necessário conhecer a logística e o seu impacto no desempenho econômico-financeiro das organizações. Assim, ao tomar conhecimento os profissionais de logística, podem diante de várias situações, desenvolver argumentos, analisar com mais precisão as diversas possibilidades, 
garantindo influência e se comunicando de forma mais clara, podendo obter o apoio necessário para gerenciar os recursos da organização (DA SILVA, 2018).

Consequentemente, os custos logísticos ocorrem à medida que as atividades realizadas para atender os clientes são executadas, com isso, há uma relação de trade-offs onde os profissionais de logística precisam conhecer e possuir a capacidade de gerenciar esta relação.

Por esse motivo, o objetivo deste trabalho foi verificar até que ponto os profissionais da área de logística conhecem e são capazes de administrar de forma eficiente os trade-offs dos custos logísticos incorridos para atendimento dos clientes. Também têm como finalidade promover o conhecimento e informações sobre um tema pouco explorado nas organizações.

Especificamente foi discutido sobre os trade-offs de custos e níveis de serviços e analisado se os profissionais de logística recebem importantes informações sobre está relação.

Inicialmente, a proliferação da logística ao ambiente corporativo de negócios, foi marcada pela fragmentação de diversas atividades por várias áreas organizacionais, o que conduziu à existência de objetivos conflitantes e à apresentação de altos custos (BALLOU, 2007). Nesta situação, a fragmentação demonstrou que o custo total ótimo só poderia ser alcançado com a integração das atividades e com a avaliação dos trade-offs pelos profissionais da área (LAMBERT e ARMITAGE, 1979; CHRISTOPHER, 1997). Nota-se que devemos considerar a logística em todas as suas faces, o desconhecimento de sua amplitude e técnicas de cortes setorizadas na tentativa de redução de custos não proporcionará os resultados esperados, podendo até incorrer em aumento de custos de outras atividades ou até mesmo desequilíbrio e perda de capacidade operacional (DA SILVA, 2018).

Em função disso, os custos totais devem ser analisados como um todo, e as estratégias levam em consideração a funcionalidade de cada atividade do setor, tendo em vista que a logística contribui de forma significativa na formação de preços e na geração de vantagens competitivas (DA SILVA, 2018). Diante disso, os processos da logística devem ser bem gerenciados, pois são responsáveis por implementar estratégias que geram qualidade e eficiência nas diversas atividades que são executadas ao longo de toda a cadeia logística, desde a origem dos produtos até o consumidor final. 
Em vista disso, vários estudos discutem a importância dos custos logísticos (MACHADO e SOUZA, 2006; LAIDENS, TELES e MULLER, 2007; KUSSANO E BATALHA, 2012; ENGBLOM et al., 2012). No entanto, trabalhos que relacionam esses custos aos níveis de serviços ainda são escassos. Informações importantes sobre custos logísticos no Brasil podem ser encontrados nos trabalhos realizados por (LIMA, 2006; GUERREIRO, BIO e MENDEL, 2011; RESENDE et al., 2012). Ainda nesta visão, trabalhos como os de (CHRISTOPHER, 1997; BIO, ROBLES e FARIA, 2002; FARIA, 2003; FELLOUS, 2009; TORRES JUNIOR e MIYAKE, 2011; TORRES JUNIOR e MIYAKE, 2011; BARBOSA, et al. 2011) que admitem a existência dos trade-offs e discutem a necessidade da sua análise e avaliação. Porém, estes estudos, apesar de fazerem uma análise ampliada de exploração dos custos logísticos, por fim, discutem os trade-offs como um tema adicional, não como principal objeto de investigação. Posto isto, se faz necessário verificar com detalhes a abordagem dos trade-offs e com isso contribuir com o avanço da literatura na área de logística. Estes estudos que abordam sobre as trocas compensatórias de custos contribuem com o desenvolvimento da logística ao evidenciar que a análise e o conhecimento dos trade-offs, é necessário para que seja encontrado o ponto de equilíbrio entre os recursos da organização e o serviço ofertado ao cliente (BUSHER e TYNDALL, 1987; DA SILVA, 2018).

Contudo, considerando o impacto dos trade-offs para o desenvolvimento das organizações e a escassez de estudos na área, e com a intenção de demonstrar a relevância de se conhecer a relação entre os custos logísticos e os níveis de serviço, justifica-se este trabalho, o qual tem como foco a seguinte problemática: Os profissionais da área de logística conhecem os trade-offs de custos e níveis de serviços logísticos? Conhecê-los possibilitará as organizações gerenciar essa relação de modo a minimizar os custos sem que haja a diminuição nos níveis de serviço aos clientes? As organizações fornecem informações logísticas e contábeis necessárias sobre a relação dos trade-offs nas diversas atividades da logística? Essas indagações revelam que o tema proposto por essa pesquisa possui grande relevância, pois o mesmo busca analisar se os profissionais conhecem a existência dos trade-offs nas atividades logística, tendo em vista o impacto econômico e financeiro para as organizações.

Com isso, destaca-se que ao conhecer os trade-offs da relação entre os custos 
e níveis de serviços, os profissionais de administração em logística poderão pensar melhor sobre os métodos atuais que estão sendo utilizados na organização, como também verificar qual técnica e/ou ferramenta pode ser utilizada nessa mensuração, servindo assim como um instrumento de gestão que poderá auxiliar as empresas na análise dos resultados dos níveis de serviço ofertados sem que haja aumento no custo total logístico.

Além disso, o conhecimento sobre esses custos contribui para que as empresas se tornem mais competitivas, visto que a eficácia da operação logística tem influência não apenas no desenvolvimento da organização, mais também no atendimento e satisfação do cliente sobre o serviço ofertado, cabendo aos profissionais de logística, determinar qual o serviço será ofertado aos clientes e quais são os recursos utilizados e/ou consumidos, para isso, é necessário que os profissionais recebam frequentemente informações contábeis e logísticas que contribuam para a tomada de decisão.

\section{FUNDAMENTAÇÃO TEÓRICA}

Historicamente, diversos autores corroboraram com a definição de serviços. Chase (1978) classificou os serviços de acordo com o grau de contato do provedor de serviço com o cliente na entrega do mesmo. Sabath (1978) fez uma importante observação, segundo o autor, o nível de serviço oferecido ao cliente deve representar o desejo do cliente e não o que a organização julga que o cliente espera, a exemplo disso, o autor, exemplifica que muitas empresas afirmam que estão ofertando certo percentual de nível de serviço, porém, nunca perguntaram ou perceberam qual o serviço e o nível os clientes desejam receber. Ainda segundo Sabath (1978) o nível de serviço ótimo é aquele que detém os clientes ao menor custo total possível. Schmenner (1986) classificou serviços como sendo uma das maneiras de aproximar serviços com operações e desafios gerenciais, podendo assim servir do ponto de apoio para o desenvolvimento da organização. Parasuraman, Zeithaml e Berry, (1988) enfatizaram que o serviço deve ter qualidade, e define como sendo a diferença entre a percepção do cliente sobre o serviço oferecido e a expectativa em relação ao que é esperado. Pereira e Cattini Junior (2001) ressaltaram que o serviço deve considerar as necessidades dos clientes, notando-se que a classificação ocorre na divisão de 
duas linhas, sendo o grau de interação e customização do serviço para o cliente.

Para completar a definição e importância do serviço para as organizações, Bowersox, Closs e Cooper (2006), apontaram que atender o nível de serviço estabelecido entre as partes constitui um dos pilares do conceito de logística integrada, sendo o nível de serviço medido em função da disponibilidade, do desempenho operacional (velocidade, consistência, flexibilidade e rapidez na recuperação de falhas) e da confiabilidade que perante o cliente está última representa qualidade da logística, ou seja, é o desempenho de todas as atividades que estão associadas ao atendimento dos pedidos e ao fornecimento das informações que são essenciais para as operações logísticas. Neste cenário, Jeffery, Butler e Malone, (2008), Da Silva (2018), recomendam que o nível de serviço ofertado pela organização é um dos fatores mais importantes para o seu sucesso e o defini como sendo o percentual de pedidos entregues dentro do prazo.

Diante disso, o nível de serviço é mensurado a medida que as necessidades dos clientes são atendidas. Nessa concepção, formular, implementar e manter uma estratégia logística, representa um dos elementos críticos para superar os desafios competitivos da atualidade (LIBERATONE e MILLER, 1995).

Por esta razão, a gestão estratégica de logística é responsável pela aplicação dos conceitos e técnicas que procuram uma solução ótima para os processos, levando em consideração a disposição de tempo e lugar, satisfazendo as expectativas dos clientes cujo ponto de equilíbrio é dado pela equação: nível de serviço ótimo versus custo total mínimo (BIO et al., 2003; DA SILVA, 2018).

Historicamente, referente aos trade-offs, Lewis e Miller (1956), foram implícitos ao destaca-lo, Lambert e Armitage (1979) foram aqueles que basearam o conceito e que corroboraram com a necessidade da sua avaliação. Como o controle dos recursos consumidos em decorrência do nível de serviço ofertado ao cliente é de responsabilidade dos profissionais da área de logística (BUSHER e TYNDALL, 1987), manifesta-se a eles a necessidade de conhecer, analisar e de avaliar os trade-offs logísticos. Entretanto, a disponibilidade, bem como a disseminação de informações contábeis apropriadas sobre os níveis de serviços e os custos logísticos também surge como requisito necessário para a avaliação dos trade-offs. Neste contexto, o cálculo dos trade-offs e a análise dos custos totais dependem de verificação, segregação e comprovação detalhada dos custos logísticos totais (BIO et al., 2002; 
FORNACIARI, PEREIRA e ZANQUETTO, 2003; WALLER e FAWCETT, 2012; DA SILVA, 2018).

Seguindo está lógica, Gimenez e Ventura (2005) salientaram que o sistema logístico é composto por vários trade-offs, como exemplo típico temos a relação do transporte com o processo de armazenagem, se a empresa cortar os custos de transporte, poderá aumentar mais custos com a armazenagem, com isso surge a necessidade de analisar todas as funções integradas umas com as outras, a fim de evitar graves erros na composição dos projetos da área de logística.

Desta forma, o conceito do custo total logístico ancora-se nas inter-relações de custos entre as atividades (MAGEE, COPACINO e ROSENFIELD, 1985). Sua apuração necessita de análise e definição dos resultados esperados da logística, em termos de serviços ao cliente, e da identificação dos custos associados para prestar esse serviço (CHRISTOPHER, 1987). Assim, os custos logísticos referem-se à forma de como essas atividades são executadas. Já os níveis de serviço estão relacionados com a qualidade com que essas atividades são gerenciadas, havendo, portanto, uma relação entre custo logístico e os níveis de serviços, pois quanto maior o nível de serviço, maior pode ser seu custo (BALLOU, 1993). No entanto, gerenciar essa relação é um dos principais desafios dos profissionais de administração em logística (LIMA, 1998). Sobre isso, Amaral e Guerreiro (2014) evidenciaram que a avaliação dos trade-offs de custos logísticos está positivamente associada à adequabilidade das informações contábeis que são fornecidas para os profissionais de logística.

Logo, a logística integrada visa estabelecer uma situação que não ocasione conflitos entre as áreas, e que favoreça tanto o atendimento aos clientes quanto a geração dos lucros. Para tanto, sabendo que a satisfação dos clientes dependia do nível de serviço ofertado e que a geração de lucros dependia do custo total incorrido na prestação do serviço, a logística integrada buscou oferecer soluções que atendessem à equação nível de serviço ótimo e custo total mínimo (FARIA e COSTA, 2005; DA SILVA, 2018). Desta maneira, o nível de serviço ofertado ao cliente representa uma importante medida de produtividade na criação das utilidades de tempo e de lugar (LAMBERT e LEWIS, 1983) e, em conjunto com o custo total, estabelece um dos pilares da logística integrada. Cada nível de serviço, por originar diferentes receitas e diferentes custos, determina qual será os lucros da organização, se nenhum serviço for ofertado, incorre-se em baixos custos, mas não se criam as 
utilidades de tempo e de lugar e não se concretizam as vendas, porém, a medida que se amplia o nível de serviço, aumentam-se os custos, mas se criam as utilidades de tempo e de lugar, estimulando positivamente as vendas (AMARAL e GUERREIRO, 2014; DA SILVA, 2018).

Em síntese, foi apresentado a plataforma teórica, que enfatiza que as decisões dentro da área de logística, devem contemplar todo o processo da cadeia de suprimentos, do início ao fim, tendo como base o conceito de que todas as atividades são interdependentes e se relacionam entre si, evidenciando o fato de que, se as atividades forem tratadas de forma isolada, o custo total logístico não será diminuído e sim elevado, sendo ainda necessário, que os profissionais de administração em logística possuam conhecimento sobre a relação de trade-offs entre custos e níveis de serviços, havendo ainda a necessidade do recebimento de informações contábeis e logísticas que cooperem para o desenvolvimento dos processos logísticos das organizações.

\section{METODOLOGIA}

Este trabalho trata-se de uma pesquisa qualitativa, pois além de descrever a complexidade do problema, também contribui para reflexão e processo de mudança do grupo em questão (RICHARDSON, 2009). Assim, a pesquisa qualitativa visa conhecer um processo que ocorre em determinado ambiente, tendo como objetivo compreendê-lo, percebê-lo e interpretá-lo (BORTONI-RICARDO, 2008).

Trata-se também, de um estudo de caso, o qual analisou de forma aprofundada um ou mais objetos, utilizando questionário para a coleta de dados (NAKANO, 2010). Ainda conforme enfatizado por Yin (2003) o estudo de caso permite a investigação de um fenômeno dentro do seu contexto na vida atual.

Este trabalho ainda pode ser classificado quanto aos fins como uma pesquisa de caráter exploratório. A principal finalidade da pesquisa exploratória é desenvolver, esclarecer e modificar conceitos.

Pesquisas exploratórias são desenvolvidas com o objetivo de proporcionar visão geral, de tipo aproximativo, acerca de determinado fato. Este tipo de pesquisa é realizado especialmente quando o tema escolhido é pouco explorado e torna-se difícil sobre ele formular hipóteses precisas e operacionalizáveis (GIL, 2008, p. 27). 
Quanto aos meios trata-se de uma pesquisa bibliográfica, realizada por meio de consulta de livros, artigos científicos, revistas, aplicação de questionário, etc., visando o aprofundamento do tema proposto na busca de sugestões e/ou soluções sobre a problemática analisada.

\subsection{Procedimentos e Delimitações da Pesquisa}

O estudo delimitou-se a uma das maiores empresas mundial no seu segmento, e obteve 34 respostas de profissionais da área de logística, onde a metodologia de pesquisa e questionário foi aplicada de modo a garantir que cada pergunta correspondesse a uma resposta e que o cruzamento de dados proporcionasse acurácia e fosse de fácil apuração, tendo como garantia, relevância e eficácia da pesquisa.

O questionário foi elaborado no google forms, e os dados foram analisados e organizados em tabelas e gráficos para possibilitar a identificação e compreensão. Conforme ressaltado por Hill e Hill (2002) os dados foram coletados seguindo metodologias éticas como explicação sobre a finalidade da pesquisa, ainda se reservando o direito de liberdade para participar. As informações das questões estruturadas foram analisadas por meio da estatística descritiva, conforme obtido nas respostas. Contudo, sobre o prisma de Martins e Theóphilo (2007) os dados das questões "livres" foram analisados por meio do diagnóstico de conteúdo, conforme Bardin (2009).

Por fim, para análise das categorias nas perguntas abertas, selecionou-se e analisou-se as respostas, cujas categorias se apresentaram recorrentes, e as perguntas fechadas foram analisadas, de modo a verificar o perfil dos profissionais da área de logística que participaram da pesquisa.

\subsection{Caracterização da Empresa}

A empresa iniciou suas operações em 1904. No ano de 2015 com algumas aquisições se tornou uma das maiores empresas mundial, líder global em fragrâncias, terceira maior empresa mundial de cosméticos, beleza, maquiagem e segunda maior em produtos voltados para o mercado profissional de cabelos. Atualmente 
comercializa seus produtos em centenas de países, através de lojas especializadas, drogarias, perfumarias, lojas de departamento, grandes varejistas e aeroportos (lojas Duty Free). Com dezenas de fábricas pelo mundo inclusive no Brasil, todas as suas operações de armazenagem, estoque, faturamento, transporte e entrega, ocorrem através de um CD (centro de distribuição) localizado na cidade de Goiânia - GO, onde foi realizado o estudo e pesquisa.

\section{RESULTADOS E DISCUSSÃO}

Após obtenção das amostras, análise e interpretação dos dados, chegou-se a categorização de temas e subtemas, conforme demonstrado a seguir.

\subsection{Perfil, Experiência Profissional e Formação Acadêmica dos Profissionais de Logística da Multinacional}

Iniciamos o estudo, identificando o perfil dos profissionais de administração em logística que participaram da pesquisa (total de 34). Dentre os resultados obtidos, além de demonstrar informações sobre a função/cargo ocupado na empresa, também foi verificado o tempo de experiência e a formação acadêmica dos profissionais.

Conforme demonstrado na Tabela 1, o resultado da pesquisa constituiu-se de: 16 (47\%) analista de logística, 10 (29\%) assistente de logística, cinco (15\%) coordenador de logística, dois (6\%) gerente de logística e um (3\%) supervisor de logística. Quanto ao tempo de trabalho na área de logística: sete (21\%) tinham até dois anos; nove (26\%) tinham de 3 a 5 anos; sete (21\%) tinham de 6 a 10 anos; nove (26\%) dos profissionais tinham de 11 a 15 anos e apenas dois (6\%) tinham mais de 16 anos de experiência na área de logística. Quanto à formação acadêmica: 47\% são formados e possui graduação superior; $41 \%$ dos profissionais de logística possuem pós-graduação (MBA/Lato Sensu); 9\% tinham apenas o ensino médio e apenas 3\% (um) possuía mestrado. 
Tabela 1 - Caracterização dos profissionais de administração em logística da Multinacional

\begin{tabular}{lcllll}
\hline \multicolumn{1}{c}{ Perfil } & $\%$ & \multicolumn{1}{c}{$\begin{array}{l}\text { Experiência } \\
\text { Profissional }\end{array}$} & $\%$ & \multicolumn{1}{c}{ Formação Acadêmica } & $\%$ \\
\hline Gerente de Logística & $6 \%$ & Mais de 16 anos & $6 \%$ & Mestrado & $3 \%$ \\
Coordenador de & 15 & & 26 & Pós-graduação (MBA/Lato & 41 \\
Logística & $\%$ & 11 a 15 anos & $\%$ & Sensu) & $\%$ \\
Supervisor de & & & 21 & & 47 \\
Logística & $3 \%$ & De 6 a 10 anos & $\%$ & Graduação/Ensino Superior & $\%$ \\
& 47 & & 26 & & $9 \%$ \\
Analista de Logística & $\%$ & De 3 a 5 anos & $\%$ & Ensino Médio & \\
Assistente de & 29 & & 21 & & \\
Logística & $\%$ & Até 2 anos & $\%$ & & \\
\hline
\end{tabular}

Fonte: os autores

Os dados obtidos demonstraram que a maioria dos profissionais de logística, são experientes e possuem cursos de graduação e especialização, o que favorece positivamente a área de logística da organização. Estudos recentes comprovaram que o conhecimento está positivamente associado à experiência profissional e a informação está positivamente associada à educação formal dos profissionais. Assim, a educação formal favorece a identificação de problemas e a eliminação de parte dos erros e dos retrabalhos, porque amplia a base de sabedoria dos profissionais (AMARAL e GUERREIRO, 2014).

Ainda nesta visão, os autores, Van Hoek, Chatham e Wilding (2002) e Hult et al., (2006) ressaltaram que a sabedoria apenas é relevante se for transformada em adequado entendimento e aplicada para a resolução de problemas, favorecendo na tomada de decisões. Diante dessa constatação, é relevante ressaltar que apenas possuir o conhecimento empírico dos trade-offs entre custos e níveis de serviços não é suficiente, é preciso aplica-lo na análise das inúmeras probabilidades de formulação das soluções logísticas (AMARAL e GUERREIRO, 2014).

Abreu e Alcântara (2014) ao analisar a importância da dimensão humana para cadeia logística verificou a necessidade de divisão em blocos: (a) competências técnicas e habilidades comportamentais, cujo somatório compõe o perfil profissional para atuar de forma eficiente na gestão de cadeias de suprimentos; e (b) estrutura organizacional arquitetada de modo a absorver esse profissional e permitir os fluxos de atividades intra e interorganizacional necessários ao bom desempenho da cadeia logística. Com isso, evidencia-se que o fator humano, sua experiência e educação formal são relevantes e contribuem para um melhor controle da cadeia logística, 
melhorando a gestão dos trade-offs de custos e níveis de serviços logísticos da multinacional.

\subsection{Integração E Conhecimento dos Profissionais de Logística sobre a Supply Chain da Multinacional}

As questões a seguir, buscou-se investigar se os profissionais de logística conheciam todos os setores e atividades que compõem a cadeia logística de suprimentos da multinacional. Todos os departamentos sinalizados no questionário da pesquisa existem e estão distribuídos da seguinte forma: o centro de distribuição localizado em Goiânia e a fábrica localizada na região metropolitana. Os dados obtidos demonstraram que 94\% (32) dos profissionais de logística sabe que existe área que trata do planejamento e gestão estratégica da cadeia de suprimentos, tendo como objetivo integrar e melhorar o fluxo entre os setores, apenas dois (6\%) apontaram que não sabem e/ou desconhece esta área.

Contudo, apesar de saber que exista área que planeja e efetua o gerenciamento da cadeia de suprimentos, os dados comprovaram que há pouco conhecimento por parte dos profissionais de logística sobre os setores e extensão total da cadeia logística.

Para qualificar o conhecimento sobre a cadeia logística da organização, optouse pelo seguinte critério: SE o percentual (\%) de respostas for $<$ (menor) que $50 \%=$ Desconhece; SE o percentual (\%) de respostas for $>$ (maior) que $50 \%$ e $<$ (menor) que $80 \%=$ Conhece parcialmente; SE o percentual (\%) for $>$ (maior) que $80 \%$ e menor que $95 \%$ = Conhece; SE o percentual (\%) de respostas for > (maior) que 95\% = Conhece totalmente toda a cadeia logística da organização, Tabela 2.

Tabela 2 - Critério utilizado para classificação sobre conhecimento da cadeia logística

\begin{tabular}{cccc}
\hline Critério & Total respostas & $\%$ Obtido & Classificação \\
\hline $\mathrm{SE}$ & $<$ (menor) & $50 \%$ & Desconhece \\
$\mathrm{SE}$ & $>$ (maior) $\mathrm{e}<$ (menor) & $>50 \% \mathrm{e}<80 \%$ & Conhece parcialmente \\
$\mathrm{SE}$ & $>$ (maior) $\mathrm{e}<$ (menor) & $>80 \% \mathrm{e}<95 \%$ & Conhece \\
$\mathrm{SE}$ & $>$ (maior) & $95 \%$ & Conhece totalmente \\
\hline
\end{tabular}

Fonte: os autores

As questões elaboradas na Tabela 3, foram de múltipla escolha, e as informações obtidas demonstraram que apenas os setores de: logística de abastecimento e Customer Service foi sinalizada por 28 (82\%) do total de 34 dos 
profissionais de logística, evidenciando scores (pontos) fraco referente ao conhecimento total da cadeia logística da multinacional.

Tabela 3 - Cadeia de suprimentos da multinacional

\begin{tabular}{|c|c|c|}
\hline Setores & $\%$ Obtido & Classificação \\
\hline Logística de Abastecimento & $82 \%$ & Conhece \\
\hline Customer Service & $82 \%$ & Conhece \\
\hline Logística de Distribuição & $79 \%$ & Conhece parcialmente \\
\hline Gerenciamento de Transportes & $74 \%$ & Conhece parcialmente \\
\hline Armazenagem & $74 \%$ & Conhece parcialmente \\
\hline Controle e Manutenção dos Estoques & $71 \%$ & Conhece parcialmente \\
\hline Área de Relação com o Cliente & $68 \%$ & Conhece parcialmente \\
\hline Logística de Fábrica & $56 \%$ & Conhece parcialmente \\
\hline Gestão de Compras e Suprimentos & $56 \%$ & Conhece parcialmente \\
\hline Planejamento de Demanda, Vendas e Produção & $56 \%$ & Conhece parcialmente \\
\hline Exportações & $56 \%$ & Conhece parcialmente \\
\hline $\begin{array}{l}\text { Gestão de Relacionamento com os } \\
\text { Fornecedores }\end{array}$ & $35 \%$ & Desconhece \\
\hline Importações & $26 \%$ & Desconhece \\
\hline Desenvolvimento de Embalagens & $24 \%$ & Desconhece \\
\hline Logística Reversa & $18 \%$ & Desconhece \\
\hline
\end{tabular}

Fonte: os autores

Considerando as metodologias funcionais internas da organização como relevantes para uma gestão integrada da cadeia logística, os estudos têm pesquisado a visão tradicional referente ao trabalho, papéis, responsabilidades e habilidades dos profissionais das áreas funcionais de logística, marketing, operações, aquisição entre outras (MURPHY E POIST, 2007; OLIVA e WATSON, 2011; KOVÁCS, TATHAM e LARSON, 2012). Na visão de Mentzer et al. (2001) os profissionais precisam possuir requisitos importantes para que esses processos ocorram, sendo, confiança, comprometimento, interdependência, compatibilidade organizacional, visão, liderança e apoio da alta gerência. Alguns autores apontam como normas comportamentais (MIN, MENTZER e LADD, 2007; ESPER, DEFEE e MENTZER, 2010; OMAR et al., 2012).

Diante desta constatação, ressalta-se a importância de conhecer toda a cadeia logística da empresa, conforme enfatizado por Miocevic e Crnjak-Karanovic (2012) as empresas para criarem valor como um processo, todos os participantes da cadeia 
precisam investir seus recursos, capacitações e conhecimentos internamente dentro da organização, como resultado, há a entrega de valor para o consumidor final. Autores como (CROXTON et al., 2001; FAWCETT, MAGNAN e MCCARTER, 2008) corroboraram com está visão ao sinalizar que este processo ocorre na esfera interna a partir da noção e do conhecimento sobre a importância da integração dos setores na organização, isso porque a efetiva integração interorganizacional, proposta fundamental para a SCM (supply chain management), depende primordialmente da capacidade das empresas em promover as relações interfuncionais entre as áreas estratégicas e que são voltadas para a criação de valor perante o consumidor.

Segundo Bio et al. (2003) qualquer nível de amplitude da cadeia em que se esteja buscando melhores soluções logísticas (a logística total da empresa, a de distribuição ou, um simples processo de alimentação de um determinado item a linha de produção), conceitualmente, as cadeias logísticas têm de ser pensadas, em função das exigências de nível de serviço a serem atendidas, sistemicamente, considerar os conjuntos de elementos e atividades interdependentes que existem para atender determinado objetivo que é definido pelo nível de serviço requerido.

Constatou-se falta de integração e conhecimento dos profissionais de logística sobre os processos internos da multinacional, demonstrando a necessidade de os profissionais logísticos buscarem conhecer as áreas funcionais e todas as atividades que compõem a cadeia logística da organização. O conhecimento da cadeia é relevante, uma vez que prevê a necessidade de integração dos fluxos internos como complementares aos externos, sendo através do conhecimento que o profissional logístico poderá agregar valor em cada processo, podendo ainda melhorar os níveis de serviço e reduzir os custos totais da cadeia logística.

\subsection{Conhecimento dos Profissionais de Logística sobre a Existência, a Relação \\ e o Impacto Econômico-Financeiro dos Trade-Offs de Custos e Níveis de Serviços Logísticos Na Organização}

A figura 1 demonstra a extensão com que os profissionais de administração em logística, analisam e avaliam o seu custo total e o nível de serviço que é ofertado aos clientes. Conforme demonstrado 56\% (19) dos profissionais apontaram que a logística influência totalmente no desenvolvimento econômico-financeiro da organização, 38\% (13) informaram que a logística influencia parcialmente e apenas 6\% (2) apontaram 
que a logística não influencia. Quanto ao custo total logístico ser determinado pelos trade-offs de custos, atribuindo as decisões sobre os processos e atividades como sendo responsáveis pela formação do custo total e da eficiência dos níveis de serviços, os profissionais demonstraram scores fracos, pois 38\% (13) sabiam totalmente, 47\% (16) sabiam parcialmente e 15\% (5) não sabiam dessa relação.

Referente à existência dos trade-offs entre custos e níveis de serviços logísticos, considerando que o aumento de custo de uma atividade pode ser compensado pelo aumento nas receitas, (82\%) dos profissionais, sendo: 14 sabiam totalmente e 14 parcialmente, apontaram saber da existência das trocas compensatórias entre custos e níveis de serviços na logística. Quanto ao entendimento de que os custos se forem tratados de forma setorizada (individualizada) não diminuem os custos podendo aumentar o custo total da cadeia logística, 26\% (9) informaram ter ciência, 50\% (17) informaram saber parcialmente e 24\% (8) informaram não ter entendimento dessa avaliação.

Desta forma, ao avaliar a relação entre níveis de serviços e custos logísticos totais, os trade-offs são ampliações dos elementos de custos que possibilitam reduções em outros elementos e/ou o acréscimo nos custos logísticos que possam propiciar aumento nas receitas, sendo este o reflexo na prestação de um melhor nível de serviço ao cliente, conduzindo a organização a melhorar seu resultado econômicofinanceiro (LAMBERT e ARMITAGE, 1979). Para Faria e Costa (2007) os trade-offs são trocas compensatórias existentes entre os custos logísticos.

No estudo, os dados obtidos, confirmam a pesquisa realizada por Amaral e Guerreiro (2014) uma vez que, ao avaliarem o conhecimento dos profissionais de logística sobre a existência dos trade-offs, notaram que a informação mais difundida é o de que a logística gera impactos na dimensão econômico-financeira das organizações, evidenciando em seu trabalho, que a maioria dos profissionais afirmaram que têm ciência, contudo, estes scores forte já era esperado tendo em vista que cada vez mais, se propaga a ideia de que o efetivo gerenciamento logístico ajuda a alavancar o desempenho econômico-financeiro das organizações (PRESUTTI e MAWHINNY, 2007; DA SILVA, 2018).

Verifica-se na pesquisa que, ao intervir nos elementos dos processos logísticos, em tempo de planejamento ou de operação, o profissional de logística deve considerar, inevitavelmente, os trade-offs (trocas compensatórias) de custos entre 
diferentes níveis de serviço e o custo da cadeia, bem como entre os elementos da cadeia, ou seja, o custo, maior ou menor, de um elemento (embalagem, transporte, armazenagem, o nível de inventários, etc.), afeta o custo de outros elementos do processo (BIO et al., 2003) influenciando o custo total da cadeia.

Figura 1 - Conhecimento dos profissionais de logística, sobre a existência dos trade-offs logísticos de custos e níveis de serviços

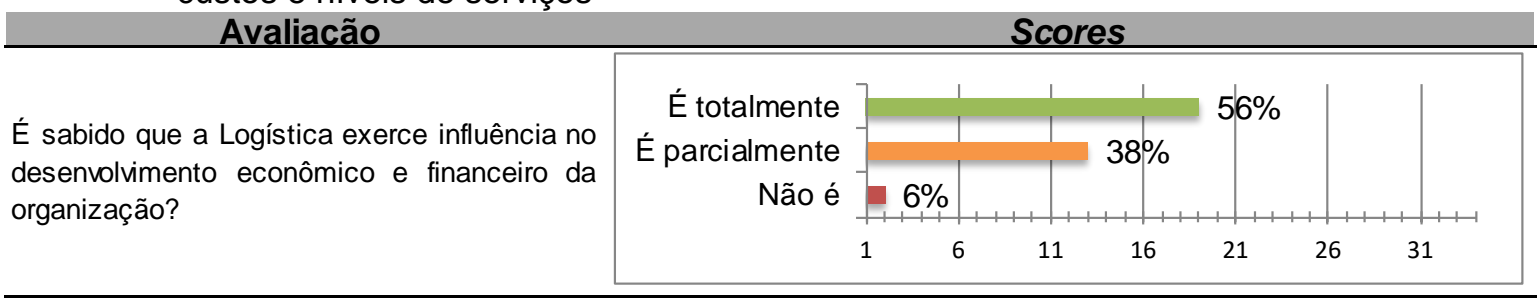

É sabido que o custo total logístico é determinado pelos trade-offs de custos, com isso as decisões sobre os processos e atividades é que são responsáveis pelos custos totais e níveis de serviços ofertados aos clientes?

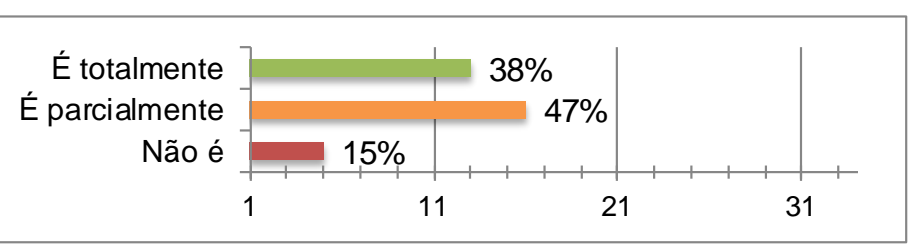

É sabido que o aumento de custo de uma atividade logística pode ser compensado pelo aumento nas receitas/vendas, evidenciando a existência de trade-offs entre custos e níveis de serviços logísticos?

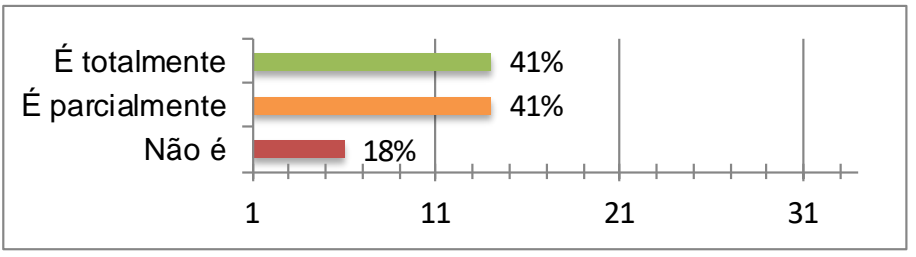

É sabido que, se os custos forem tratados de forma individualizada, podem não diminuir e sim aumentar o custo total na cadeia logística?

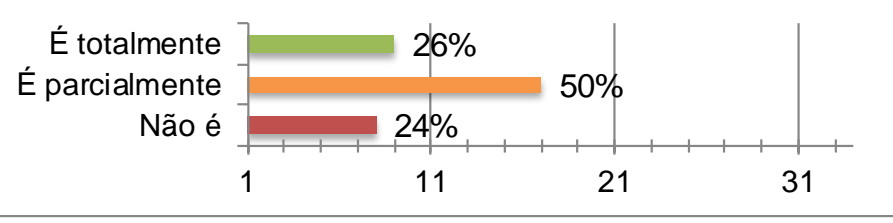

É sabido que a redução de custo em uma atividade pode não gerar resultados positivos para a organização? Exemplo 1: Ao reduzir 2\% no custo total de frete, houve diminuição do nível de serviço, impactando de forma negativa em $10 \%$ nas vendas.

Exemplo 2: A empresa decidiu diminuir a armazenagem do $C D$, com isso possui total conhecimento que será necessário de alguma forma aumentar o número com transportes para abastecimento

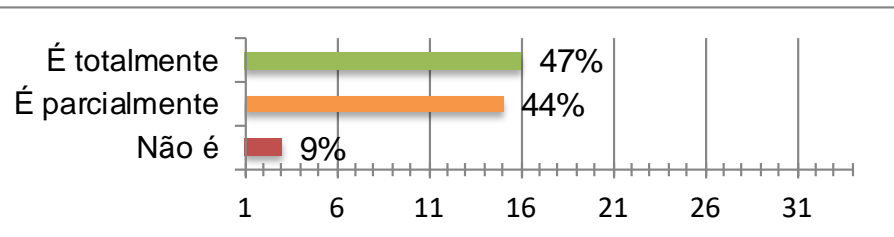
do CD.

O custo incorrido nas atividades logísticas da organização, se justifica perante o nível de serviço prestado ao cliente (de alguma forma é realizado este tipo de avaliação)?

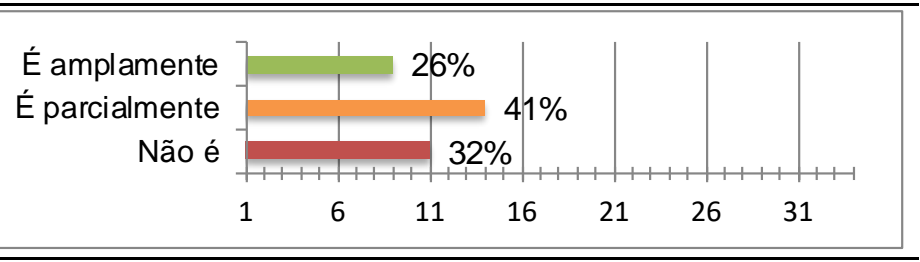

Fonte: os autores 
Diante dos dados, constata-se que os profissionais de administração logística da multinacional sabem da existência dos trade-offs entre custo e níveis de serviços, porém, conforme observado por Amaral e Guerreiro (2014) os resultados indicam que o conhecimento dos trade-offs ainda são limitados, pois evidenciam que os profissionais da área de logística sabem que as trocas compensatórias existem, mas não têm um entendimento claro sobre o seu conceito e do seu funcionamento.

Destaca-se que, ao tomar conhecimento da existência dos trade-offs de custos e níveis de serviços, o profissional de logística, poderá reduzir custos, podendo ainda melhorar os níveis de serviços através do planejamento e controle dos processos, contribuindo para o desenvolvimento da organização, as quais buscam os menores custos e níveis de serviços mais eficazes.

\subsection{Relevância das Informações Contábeis e Logísticas para os Profissionais de Administração em Logística}

Foi analisado se os profissionais da área de logística recebiam informações contábeis e logísticas que ajudavam na tomada de decisão. Solicitou-se para atribuir scores (pontos), seguindo o critério a seguir: de 0 a 2 pontos = informações não ajudam; 0 a 4 pontos = ajudam parcialmente e 5 pontos = ajudam completamente.

Verificou-se que apesar da multinacional possuir sistemas de informações apurados, como, DRE, Return on Investiment (ROI), análises de despesas, acompanhamento orçamentário, sistemas de gestão e acompanhamento de pedidos, etc.), voltados para a mensuração de custos e dos níveis de serviços, as respostas obtidas demonstraram que dos 34 profissionais da área de logística, considerando uma média geral das respostas, somente 50\% (17) dos profissionais informaram que recebem informações que ajudam completamente a verificar a relação de trade-offs entre custos e níveis de serviços e que as informações obtidas cooperam com a formulação de estratégias que contribuem para o desenvolvimento logístico da organização (Tabela 4).

Diante disso, renomados autores (LAMBERT, STOCK e VANTINE, 1998; BALLOU, 2001; BOWERSOX e CLOSS, 2001) apontaram sobre este problema, advertindo que, em essência, esta inadequação e falta de informações contábeis e logísticas, dificultam, sobremaneira, as empresas em potencializar seus resultados, 
tendo em vista que essas informações são relevantes para a tomada de decisão e melhoria dos processos da área de logística.

Ressalta-se que os profissionais da área de logística são tomadores de decisões, pois lidam com amplo leque de disponibilidades (opões) para otimizar o resultado econômico-financeiro das empresas.

Para conseguir melhorar os processos, é necessário que os departamentos que acompanham os resultados empresariais, com seus diversos sistemas de custos e mensuração dos níveis de serviços forneçam para os profissionais de logística, informações adequadas e adaptadas ás suas necessidades, buscando atender ao seu processo de raciocínio e decisão, no conteúdo, grau de detalhe, periodicidade, enfim, nos atributos que caracterizam uma informação gerencial eficaz, (BIO et al., 2003; DA SILVA, 2018).

Tabela 4 - Informações contábeis e logísticas

Informações

Respostas (scores)

0 a 2 (não) 3 a 4 (parcialmente) 5 (completamente)

\begin{tabular}{|c|c|c|c|}
\hline $\begin{array}{lccc}\text { Ajudam e } & \text { contribuem } & \text { com } & \text { o } \\
\text { desenvolvimento da área de logística? }\end{array}$ & $3 \%$ & $44 \%$ & $53 \%$ \\
\hline $\begin{array}{l}\text { Ajudam a verificar e apurar as trocas } \\
\text { compensatórias (trade-offs) de custos } \\
\text { necessários para a mensuração do custo } \\
\text { total logístico? }\end{array}$ & $6 \%$ & $50 \%$ & $44 \%$ \\
\hline $\begin{array}{l}\text { Ajudam a formular estratégias que } \\
\text { contribuem para o melhoramento da } \\
\text { logística? }\end{array}$ & $3 \%$ & $44 \%$ & $53 \%$ \\
\hline $\begin{array}{l}\text { Ajudam a mensurar, analisar e gerenciar a } \\
\text { relação existente (trade-offs) entre custos e } \\
\text { níveis de serviços logísticos da } \\
\text { organização? }\end{array}$ & $6 \%$ & $44 \%$ & $50 \%$ \\
\hline
\end{tabular}

Fonte: os autores.

Nota. Informações Recebida das Áreas de Customer Service, Controles e Custos, Controladoria, Contabilidade Gerencial e Financeira, Auditoria e Planejamento Estratégico da Logística.

Diante dos resultados, comprovou-se que há um paradigma a ser quebrado, os departamentos que possuem informações sobre os custos e níveis de serviços encaram a logística apenas como uma função operacional sendo administrado por meio de acompanhamento de um orçamento de despesas departamentais, com isso muitos profissionais envolvidos nas operações logísticas não recebem devidamente as informações contábeis necessárias para avaliação dos resultados (BIO et al., 2003) é o que se confirmou no estudo de caso através das respostas obtidas. 
Afinal, no estudo de caso, corrobora-se com a crítica dos renomados autores de logística, ao confirmar-se que, em realidade, e ainda na prática, evidencia-se a falta de informações contábeis e logísticas adequadas e suficientes para a tomada de decisão e gestão estratégica da cadeia logística da organização. Isso pode fazer com que, inadvertidamente, a pressão por cortes individuais de custos não seja percebida como um equívoco (AMARAL e GUERREIRO, 2014; DA SILVA, 2018).

Por fim, ressalta-se sobre a relevância dos setores de controladoria, contabilidade e planejamento estratégico, apoiarem os profissionais de administração logística através de informações que auxiliarão na tomada de decisão e análise apurada dos trade-offs de custos e níveis de serviços logístico, o que contribui positivamente para a eficiência da área e o desenvolvimento econômico e financeiro da organização.

\section{CONSIDERAÇÕES FINAIS}

Após análise e demonstração dos resultados obtidos, verifica-se a necessidade de conhecer os trade-offs logísticos relacionados aos objetivos de desempenho entre os custos e os níveis de serviços das atividades da área de logística, cada processo pode oferecer uma troca compensatória diferente e que se for analisado de forma instantânea, ou seja, de forma individualizada, poderá apresentar nenhuma vantagem ou resultado positivo, porém, o resultado geral da cadeia, certamente apresentará maximização nos serviços e minimização no custo total envolvido para atender os clientes.

Com isso, confirma-se a crítica de renomados autores de logística, no estudo de caso, pois evidencia-se a falta de informações contábeis e logísticas adequadas e suficientes para a gestão estratégica da cadeia logística da multinacional. Comprovase também que há falta de conhecimento por parte dos profissionais de logística em entender o conhecimento e a funcionalidade dos trade-offs existente nas atividades logísticas, tendo em vista que essas trocas compensatórias são responsáveis por determinar o custo total e o nível de serviço ofertado.

Enfim, conclui-se que a maioria dos profissionais logísticos sabem da existência dos trade-offs, tendo ciência que o aumento de um custo pode ser justificado pelo aumento nas vendas, gerando-se assim receita e não somente um custo, no entanto, 
se faz necessário um maior entendimento e funcionamento de como as trocas compensatórias estão relacionadas com os custos e níveis de serviços, destacandose que os processos por serem interdependentes devem ser analisados cuidadosamente, pois cada atividade pode gerar um resultado (custos e níveis de serviço) diferentes para a organização.

Além das sugestões apresentadas neste artigo, recomenda-se que novas investigações deem continuidade a este estudo e explorem um maior número de especificidades e busquem um aprofundamento sobre os trade-offs, buscando analisar por exemplo, quais trade-offs são mais relevantes, mais conhecidos, mais avaliados e quais podem impactar sobremaneira os custos e níveis de serviços empresariais.

\section{REFERÊNCIAS}

ABREU, A.; ALCANTARA, R. L. C. A gestão de pessoas e a estrutura organizacional sob a perspectiva da cadeia de suprimentos. Reuna, Belo Horizonte - MG, Brasil, v.19, n.1, p. 6788, 2014.

AMARAL, J. V.; GUERREIRO. R. Conhecimento e Avaliação dos Trade-offs de Custos Logísticos: um Estudo com Profissionais Brasileiros. Revista Contabilidade \& Finanças USP, São Paulo, v. 25, n. 65, p. 111-123, 2014.

BALLOU, R. H. Logística empresarial: transportes, administração de materiais e distribuição física. São Paulo, Editora Atlas, 1993. 388 p.

BALLOU, R. H. Gerenciamento da cadeia de suprimentos: planejamento, organização e logística empresarial. 4. ed. Porto Alegre: Bookman, 2001. 532 p.

BALLOU, R. H. Gerenciamento da cadeia de suprimentos: logística empresarial. 5. ed. Porto Alegre: Bookman, 2006. 616 p.

BALLOU, R, H. The evolution and future of logistics and supply chain management.

European Business Review. Bradford, v.19, n. 04, p. 332-348, 2007.

https://doi.org/10.1108/09555340710760152

BARDIN, L. Análise de conteúdo. 5. ed. Lisboa, Portugal, 2008. 281 p.

BARBOSA ET AL. A pesquisa em trade-offs de custos logísticos: estudo bibliométrico no período de 2006 a 2016. Revista Produção Online. Florianópolis, SC, v. 18, n. 2, p. 641664, 2018. DOI: http://dx.doi.org/10.14488/1676-1901.v18i2.2882

BIO, S. R.; ROBLES, L. T.; FARIA, A. C. Em busca da vantagem competitiva: trade-offs de custos logísticos em cadeias de suprimentos. Revista de Contabilidade CRC-USP, 6 (19), 5-18, 2002. 
BIO, S. R.; ROBLES, L. T.; FARIA, A. N. O papel da controladoria no apoio às decisões logísticas: um estudo de caso. In: CONGRESSO BRASILEIRO DE CUSTOS, X., 2003, Guarapari. Anais... Guarapari: Associação Brasileira de custos, 2003. Disponível em: https://anaiscbc.emnuvens.com.br/anais/article/view/2497/2497. Acesso em: 6 jan. 2017.

BOWERSOX, D. J.; CLOSS, D. J. Logística empresarial: o processo de integração da cadeia de suprimento. São Paulo: Editora Atlas, 2001. 594 p.

BOWERSOX, D. J.; CLOSS, D J.; COOPER, M. B. Gestão logística de cadeias de suprimentos. Porto Alegre: Bookmam, 2006. 528 p.

BUSHER, J. R.; TYNDAL, G.R. Logístics excellence. Management accounting. Montvale, v. 69 , n. 02 , p. $32-39,08 / 1987$.

CHASE, R. B., Where does the customer fit into the service operation, Harvard Business Review, v. 56, n. 6, p. 137-142, 1978.

CHRISTOPHER, M. Assessing the costs of logistics service. Working paper, cranfield school of management, Working Paper, p. 61 - 87, 1987.

CHRISTOPHER, M. Logística e gerenciamento da cadeia de suprimentos: estratégia para a redução de custos e melhoria dos serviços. São Paulo: Pioneira. 1997. 240 p.

CROXTON, K. L.; GARCIA-DASTUGUE, S. J.; LAMBERT, D. M.; ROGERS, D. S. The supply chain management process. The International Journal of Logistics Management, v.12, n.2, p.13-36, 2001. https://doi.org/10.1108/09574090110806271

DA SILVA, J. A. B. Customer service logistics como estratégia organizacional: um estudo de caso em uma multinacional. Brazilian Journal of Production Engineering, v. 4, n. 4, p. 226-244, 2018.

DA SILVA, J. A. B.; SILVA, S. Critérios de qualidade em serviços de transporte público urbano: uma contribuição teórica. Brazilian Journal of Production Engineering, v. 4, n. 1, p. 83-98. 2018.

DA SILVA, J. A. B.; SCHLAG, F. Aspectos tangíveis e intangíveis na definição de qualidade do transporte público. In: ANAIS DO SEMINÁRIO DE PESQUISA, PÓS-GRADUAÇÃO, ENSINO E EXTENSÃO DO CAMPUS ANÁPOLIS DE CSEH (SEPE), 3, n. 1. Anais... 2017.

ESPER, T. L.; DEFEE, C. C.; MENTZER, J. T. A framework of supply chain orientation. The International Journal of Logistics Management, v. 21, n. 2, p.161-179, 2010. https://doi.org/10.1108/09574091011071906

FARIA, A. C. Custos logísticos: uma abordagem na adequação das informações de controladoria a gestão da logística empresarial. 2003. 313 f. Tese (Doutorado em Ciências Contábeis) - Programa de Pós-graduação em Ciências Contábeis, Departamento de Contabilidade e Atuária, Faculdade de Economia, Administração e Contabilidade da Universidade de São Paulo, 2003.

FARIA, A. C.; COSTA, M. F. G. Gestão de custos logísticos: custeio baseado em atividades (ABC), balanced scorecard (BSC), valor econômico agregado (EVA). 1 ed. São Paulo: Atlas, 2005. 448 p.

FARIA, A. C.; COSTA, M. F. G. Gestão de custos logísticos: custeio baseado em 
atividades (ABC), balanced scorecard (BSC), valor econômico agregado (EVA). 1 ed. 2 reimpressão. São Paulo: Atlas, 2007. 431 p.

FAWCETT, S. E.; MAGNAN, G. M.; MCCARTER, M. W. Benefits, barriers and bridges to effective supply chain management. Supply Chain Management: An International Journal, v.13, n.1, p. 35-48, 2008.

FELLOUS, S. M. Gestão da cadeia de suprimentos no Brasil e a utilização de instrumentos da contabilidade gerencial: uma avaliação sob a perspectiva dos profissionais envolvidos, 2009. 218 f. Dissertação (Mestrado em Ciências Contábeis) Faculdade de Economia, Administração e Contabilidade, Programa de Pós-Graduação em Ciências Contábeis, São Paulo, SP, Brasil. 2009.

FORNACIARI, G.; PEREIRA, M. M. A. M.; ZANQUETTO FILHO, H. A necessidade de segregação e evidenciação dos custos logísticos nos relatórios contábeis. In: CONGRESO INTERNACIONAL DE COSTOS, VIII., 2003, Punta del Este. Anais... Uruguai: Asociación Uruguaya de Costos, 2003. Disponível em:

http://www.intercostos.org/index.php/pt/component/congresos/791?view=documento. Acesso em: 15 jan. 2017.

GIL, A. C. Métodos e técnicas de pesquisa social. 6. ed. São Paulo: Atlas, 2008, p. 27. $200 \mathrm{p}$.

GIMENEZ, C.; VENTURA, E. Logistics production, logistics marketing and external integration. International Journal of Operations \& Production Management, v.25, n.1, p.20-38, 2005. https://doi.org/10.1108/01443570510572222

GUERREIRO, R.; BIO, S. R.; MENDEL, S. F. Logística integrada, gestão da cadeia de suprimentos e mensuração de custos e resultados logísticos: um estudo com empresas brasileiras. Advances in Scientific and Applied Accounting. São Paulo, v. 4, n.1, p.73100, 2011.

HILL, M.; HILL, A. Investigação por questionário. 2 ed. Lisboa, Portugal: Edições Sílabo, 2002.

HULT, G. T. M.; KETCHEN JR., D. J.; CAVUSGIL, S. T.; CALANTONE, R. J. Knowledge as a strategic resource in supply chains. Journal of Operations Management, v. 24, n. 5, 458475, 2006. https://doi.org/10.1016/i.jom.2005.11.009

JEFFERY, M. M.; BUTLER, R. J.; MALONE, L. C. Determining a cost-effective customer service level. Supply Chain Management: An International Journal, v. 13, n. 3, p. 225-232, 2008. https://doi.org/10.1108/13598540810871262

KOVÁCS, G.; TATHAM, P.; LARSON, P. D. What skills are needed to be a humanitarian logistician? Journal of Business Logistics, v. 33, n. 3, p. 245-258, 2012. https://doi.org/10.1111/j.2158-1592.2012.01054.x

KUSSANO, M. R.; BATALHA, M. O. Custos Logísticos Agroindustriais: avaliação do escoamento da soja em grão do Mato Grosso para o mercado externo. Gestão \& Produção. São Carlos, v. 19, n. 3, p. 619-632, 2012.

LAIDENS, G.; TELES, C. D.; MULLER, C. J. Avaliação dos custos logísticos em empresas do setor alimentício. Estudos tecnológicos, v. 3, n. 2 p. 83-91, 2007. 
LALONDE, B. J. Integrated distribution systems: a management perspective. International Jounal of Physical Distribution \& Logistics Management. Bradford, v. 23, n. 05, p. 04-12, 1993.

LAMBERT, D. M.; ARMITAGE, H. M. Distribuition Costs: the challenge: The key to managing the physical distribution function is total cost analystis, rather than haphazard stabs at cutting specific costs. Magement Accouting (pre-1986). Montvale, v. 60, n. 11, p. 33-37, 45, 05/1979.

LAMBERT, D. M.; LEWIS, C. M. Managing customer service to build market share and increase profit. Business Quarterly. London, v. 48, n. 03, p. 50-57, 10/1983.

LEWIS, H. T.; CULLITON, J. W. Characteristiics of air freight and its market. in. the of air freight in phyfical distribution. Boston: Division of Research Graduate School of Business Administration, Havard University, 1956.

LIBERATONE, M. J.; MILLER, T. C. A decision support appoach for transport Carrier and mode selection. Journal of Business Logistics. Hoboken, v. 16, n. 02, p. 85-115, 1995.

LIMA, M. P. Custos logísticos: uma visão gerencial. 1998. Disponível em:

http://www.ilos.com.br/web/custos-logisticos-uma-visao-gerencial/. Acesso em: 03 jan. 2018.

LIMA, M. P. Custos logísticos na economia brasileira. Revista Tecnologística, 2006. Disponível em: http://www.ilos.com.br/web/custos-logisticos-na-economia-brasileiral. Acesso em: 10 jan. 2017.

MAGEE, J. F.; COPACINO, W. C.; ROSENFIELD, D. B. Modern Logistics Managment: Integrting Marketing, Manufacturing, and Physical Distribution. New York, John Wiley \& Sons, 1985.

MARTINS, G. A. THEÓPHILO, C. R. Metodologia da investigação científica para ciências sociais aplicadas. São Paulo; Atlas, 2009. 247 p.

MENTZER, J. T.; FLINT, D. J.; HULT, T. M. Logistics service quality as a segmentcustomized process. Journal of Marketing. Chicago, v. 65, n. 04, p. 82-104, 2001. https://doi.org/10.1509/jmkg.65.4.82.18390

MIOCEVIC, D.; CRNJAK-KARANOVIC, B. The mediating role of key supplier relationship management practices on supply chain orientation: The organizational buying effectiveness link. Industrial Marketing Management, v. 41, p.115-124, 2012.

https://doi.org/10.1016/j.indmarman.2011.11.015

NAKANO, D. Métodos de pesquisa adotados na engenharia de produção e gestão de operações. In: CAUCHICK, P. M. Metodologia de pesquisa em engenharia de produção e gestão de operações. 2 ed. Rio de Janeiro: Elsevier, 2011. 280 p.

OLIVA, R.; WATSON, N. Cross-functional alignment in supply chain planning: a case study of sales an operations planning. Journal of Operations Management, v. 29, p.434-448, 2011. https://doi.org/10.1016/j.jom.2010.11.012

OMAR, A.; DAVIS-SRAMEK, B.; FUGATE, B. S.; MENTZER, J. T. Exploring the complex social processes of organizational change: supply chain orientation from a manager's perspective. Journal of Business Logistics, v. 33, n.1, p. 4-19, 2012.

https://doi.org/10.1111/j.0000-0000.2011.01034.x 
PARASURAMAN, A.; ZEITHAML, V. A.; BERRY, L. L. SERVQUAL: A multiple-Item Scale for Measuring Consumer Perceptions of Service Quality. Journal of Retailing, v.64, n. 1, p. 1240, 1988.

PEREIRA, S. C. F; CATTINI JUNIOR, O. A model of service classification based on consumer needs cliente. In: Twelfth Annual Conference of the Production and Operations Management Society, POMS-2001, march 30-April 2, Orlando FI, 2001.

PRESUTTI, W. D.; MAWHINNEY, J. R. The supply chain-finance link. Supply Chain Management Review. Framington, v. 11, n. 06, p. 32-38, 2007.

RESENDE, P.; SOUZA, P. R.; MONTEIRO, P. R. R.; DIAS, B. C.; SILVA, B. R. O.; SCOTT, F. A.; SOUZA, M. P. G. Fundação Dom Cabral, Pesquisa de custos logísticos no Brasil, 2012. Disponível em:

<http://acervo.ci.fdc.org.br/AcervoDigital/Relat\%C3\%B3rios\%20de\%20Pesquisa/Relat\%C3\% B3rio\%20de\%20Pesquisa\%202012/Relat\%C3\%B3rio\%20Pesquisa\%20Custo\%20Log\%C3 \%ADstico\%20no\%20Brasil.pdf> Acesso em: 11 jan. 2017.

SABATH, R. E. How much service do customers really want? Business Horizons. Greenwich, v. 21, n. 02, p. 26-32, 04/1978.

SCHMENNER, R. W., How can service businesses survive and prosper. Sloan Management Review, v. 27, n. 3, p. 21-32, 1986.

TORRES JUNIOR, N.; MIYAKE, I. D. Melhoria de desempenho em serviços: alternativas para lidar com o trade-off entre eficiência e eficácia. Revista Produção Online, v.11, n.1, mar. 2011. DOI: https://doi.org/10.14488/1676-1901.v11i1.539

VAN HOEK, R. I.; CHATHAM, C.; WILDING, R. Managers in supply chain management, the critical dimension. Supply Chain Management, v. 7, n. 3, p. 119-125, 2002.

https://doi.org/10.1108/13598540210436568

YIN, R. K. Estudo de caso: planejamento e métodos. 3. ed. Porto Alegre: Bookman, 2003. 211p.

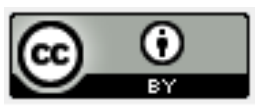

Artigo recebido em: 25/02/2018 e aceito para publicação em: 17/02/2019

DOI: http://dx.doi.org/10.14488/1676-1901.v19i1.3176 